\title{
Melting and structure of the vortex solid in strongly anisotropic layered superconductors with random columnar pins
}

\author{
Chandan Dasgupta* \\ Department of Physics, Indian Institute of Science, Bangalore 560012, India \\ Oriol T. Valls ${ }^{\dagger}$ \\ School of Physics and Astronomy and Minnesota Supercomputer Institute, University of Minnesota, \\ Minneapolis, Minnesota 55455, USA
}

\begin{abstract}
We study the melting transition of the low-temperature vortex solid in strongly anisotropic layered superconductors with a concentration of random columnar pinning centers small enough so that the areal density of the pins is much less than that of the vortex lines. Both the external magnetic field and the columnar pins are assumed to be oriented perpendicular to the layers. Our method, involving numerical minimization of a model free energy functional, yields not only the free energy values at the local minima of the functional but also the detailed density distribution of the system at each minimum: this allows us to study in detail the structure of the different phases. We find that at these pin concentrations and low temperatures, the thermodynamically stable state is a topologically ordered Bragg glass. This nearly crystalline state melts into an interstitial liquid (a liquid in which a small fraction of vortex lines remain localized at the pinning centers) in two steps, so that the Bragg glass and the liquid are separated by a narrow phase that we identify from analysis of its density structure as a polycrystalline Bose glass. Both the Bragg glass to Bose glass and the Bose glass to interstitial liquid transitions are first order. We also find that a local melting temperature defined using a criterion based on the degree of localization of the vortex lines exhibits spatial variations similar to those observed in recent experiments.
\end{abstract}

\section{INTRODUCTION}

The mixed phase of type-II superconductors with random pinning is generally regarded to be an archetypal test system for the study of the effects of quenched disorder on the structure and melting of solids. In this phase, magnetic flux penetrates the sample as quantized vortex lines which form a special physical system known as "vortex matter." The fascinating equilibrium and dynamical properties of vortex matter in the mixed phase of high-temperature superconductors (HTSC's) have prompted considerable experimental and theoretical attention for more than a decade (see Ref. 1 for an early review). The mixed phase of HTSC's is strongly affected by the presence of pinning centers, either intrinsic or artificially generated. Understanding the effects of pinning in these systems is basic for practical applications because pinning strongly influences the value of the critical current. ${ }^{2}$

The vortex lines in a pure type-II superconductor form a triangular lattice (the Abrikosov lattice) at low temperatures. Because of enhanced thermal fluctuations in highly anisotropic, layered HTSC's, the Abrikosov lattice in very pure samples, in a magnetic field perpendicular to the layers (the field will be assumed to be in this direction throughout our discussion), undergoes a first-order melting transition ${ }^{1}$ into a resistive vortex liquid (VL) as the temperature $T$ increases. Random pinning destroys the long-range translational order of the Abrikosov lattice and leads to the occurrence of a variety of glassy phases at low $T$. It is now generally accepted that in systems with random point pinning, a topologically ordered low-temperature phase with quasi-long- range translational order [denoted as the "Bragg glass" (BrG) phase] occurs at low fields if the pinning disorder is sufficiently weak. This has been established theoretically ${ }^{3-5}$ as well as experimentally (see, e.g., Ref. 6). The possibility of an amorphous vortex glass (VG) phase, with nonlinear voltage-current characteristics and vanishing resistance in the zero-current limit, in systems with strong pinning (or at high magnetic fields where the effects of pinning disorder are enhanced) was suggested by Fisher ${ }^{7}$ (see also Ref. 8). However, in spite of extensive investigations, the existence of a true VG phase (i.e., an amorphous glassy phase thermodynamically distinct from the high-temperature VL) in systems with uncorrelated point pinning remains very controversial: different calculations ${ }^{9,10}$ lead to different conclusions and the experimental situation ${ }^{11,12}$ is similarly contradictory. A variety of "glassy" behavior has been reported in different experiments (see Ref. 13 and references therein) near the firstorder melting transition of the $\mathrm{BrG}$ phase of both conventional superconductors and HTSC's with point pinning. It has been suggested ${ }^{13,14}$ that these observations may be understood if it is assumed that the melting of the $\mathrm{BrG}$ phase occurs in two steps: the BrG first transforms into a "multidomain" glassy phase that melts into the usual VL at a slightly higher $T$.

Columnar pinning defects can be produced, for example, as damage tracks arising from heavy-ion bombardment. The technological importance of these extended defects oriented parallel to the direction of the external magnetic field has been long recognized: ${ }^{15,16}$ they are highly effective in increasing the critical current by localizing vortex lines along 
their length. Heavy-ion irradiation produces a random array of parallel columnar defects each of which can trap one or more vortices at low temperatures. The effects of such random arrays of extended defects, oriented perpendicular to the superconducting layers, on the properties of the mixed phase of HTSC's have been extensively studied experimentally. ${ }^{17-19}$ The same question has also been examined theoretically ${ }^{20-23}$ and numerically. ${ }^{24-27}$ When the columnar pinning is strong, and their concentration exceeds that of the vortex lines, a so-called "strong" Bose glass (BoG) phase with nearly all the vortex lines localized at the pinning centers occurs ${ }^{20,24}$ at low $T$. This phase is strongly disordered with very-short-range translational and bond-orientational correlations. The behavior in this regime is fairly well understood in terms of a mapping ${ }^{20}$ of the thermodynamics of the system to the quantum-mechanical properties of a twodimensional system of interacting bosons in a random potential.

Much less is known about the behavior in the dilute pin limit where the concentration of pins is much smaller than that of vortex lines. In such systems, one expects ${ }^{21}$ a "weak" BoG phase at low temperatures, with a small fraction of vortex lines localized strongly at the pinning centers and the remaining ones localized relatively weakly in the interstitial region between pinning centers. As the temperature is increased, this phase should ${ }^{21,23}$ melt into an interstitial liquid (IL). In the IL phase, some of the vortices remain trapped at the pinning centers, while the other, interstitial ones, form a liquid. The pinned vortices are expected ${ }^{21,23}$ to delocalize, thereby forming the usual VL, at a crossover occurring at a higher temperature. The melting transition of the "weak" BoG phase into the IL is predicted ${ }^{21,22}$ to be first order for small pin concentrations, whereas the "strong" BoG to VL transition occurring for large pin concentrations is known ${ }^{20,22}$ to be a continuous one.

A first-order melting transition of the "weak" BoG phase has been observed ${ }^{19}$ in recent experiments on samples of $\mathrm{Bi}_{2} \mathrm{Sr}_{2} \mathrm{CaCu}_{2} \mathrm{O}_{8+\mathrm{x}}$ (BSCCO) with a small concentration of columnar pins. The BoG phase in these systems is found ${ }^{18,19}$ to have a polycrystalline structure with ordered vortex crystallites of different orientations embedded in the interstitial region between vortices pinned at the columnar defects. If the pin concentration is sufficiently small, the melting of this BoG phase upon increasing the temperature occurs into an IL phase with a fraction of vortex lines remaining pinned at the defects. These pinned vortices delocalize at a higher temperature. ${ }^{18}$ The temperature at which the first-order BoG to IL transition occurs is ${ }^{17,18}$ very close to the melting temperature of the same system without the columnar pins, if the pin concentration is small. As the pin concentration is increased, the melting temperature increases and the transition eventually becomes continuous. The difference between the melting temperature and the temperature at which the pinned vortices in the IL phase delocalizes decreases and eventually goes to zero as the pin concentration increases. Another interesting feature, found experimentally for both point ${ }^{28}$ and columnar ${ }^{18}$ pinning, is that the melting of the low $T$ "solid" phase is "inhomogeneous" in that it occurs locally over a range of temperatures: the local transition temperature, which can be measured from the local magnetization, is dif- ferent in different regions of the sample. This inhomogeneity of the local melting temperature is believed ${ }^{28}$ to be closely related to the local arrangement of the specific pinning centers (the so-called "pinning landscape") in each sample studied.

The polycrystalline nature of the BoG phase for small pin concentrations has also been observed in simulations. ${ }^{25} \mathrm{Re}$ cent numerical work ${ }^{27}$ indicates that a BrG phase, with topological order, may also exist in such systems provided that the pin concentration is sufficiently small. In these simulations, the BrG phase is found to melt into the IL phase via a first-order transition as the temperature is increased. As the pin concentration is increased beyond a critical value, the BrG phase disappears ${ }^{27}$ and a low-temperature BoG phase with a continuous BoG to IL transition upon increasing the temperature is found. ${ }^{26,27}$

In this paper, we approach these problems through a different numerical method. We consider a layered, strongly anisotropic superconductor (such as BSCCO) with a dilute random array of columnar defects oriented perpendicular to the layers, and with a magnetic field applied parallel to the columnar pins. We describe the equilibrium properties of this system in terms of vortex density variables, using a free energy functional of the Ramakrishnan-Yussouff ${ }^{29}$ (RY) form. We numerically minimize a spatially discretized version of this free energy functional and obtain the vortex density configuration at each local minimum. Analysis of these density configurations, both in terms of a variety of correlation functions and by direct visualization of the arrangement of the vortices (vortex positions are identified with those of local peaks of the vortex density), allows us to identify the nature of the phases corresponding to different local minima of the free energy. Comparison of the values of the free energy at the minima as the temperature varies yields the transition temperatures. A similar procedure has been successfully employed for the case of a regular array ${ }^{30,31}$ of columnar pinning centers.

We find in our study that a Bragg glass phase exists at low temperatures for samples with a small concentration of columnar pins. We will present evidence for this phase from our analysis of the vortex density and associated correlation functions. Upon warming, this phase melts into what we show to be an interstitial liquid (IL) phase, but the melting is shown ${ }^{32}$ to occur in two steps: the BrG and IL phases are separated, over a narrow temperature range, by an intermediate phase that exhibits a multidomain polycrystalline structure. We show that this intermediate phase should be identified as a BoG. The temperature of the upper (BoG to IL) transition is approximately independent of the columnar pin concentration $c$, at the low $c$ values studied. Both the $\mathrm{BrG}$ to $\mathrm{BoG}$ and the BoG to IL transitions are found to be first order. We also find that a local melting temperature can be suitably defined using a criterion based on the degree of localization of the vortices, and that its behavior (in particular, its spatial variation) is quite consistent with what is seen in experiments. ${ }^{18,28}$ The value of the local melting temperature is strongly correlated with the presence of topological defects (dislocations) in the vortex solid, which, in turn, is correlated with the local arrangement of the pinning centers. We also show that the transition to the IL phase corresponds to a 
percolation of regions containing liquidlike (delocalized) vortices across the sample.

After this Introduction, we discuss our definitions, model, and numerical methods in the next section. Then in Sec. III we present our results. We discuss first the free energy minima and their study through the use of tools such as correlation functions, local peak-density plots, and Voronoi construction for the lattice formed by local density peaks. These tools allow the identification of the phases at each free energy minimum, as we shall show. Then we derive the phase diagram and show that indeed the BrG and IL phases are separated by a thin sliver of BoG. Finally, our results for the nature of the local melting are displayed and discussed. A brief conclusions section recapitulates the main points of the paper and discusses them in the context of existing results.

\section{METHODS}

The general procedures that we use are quite similar to those employed in previous work on a regular array of columnar pins. ${ }^{30,31} \mathrm{We}$ will therefore give here only a brief summary, emphasizing the details that are different in the random case considered here.

The system we study is a layered superconductor in the extreme anisotropic limit, that is, with vanishing Josephson coupling between layers, which are then coupled via the electromagnetic interaction only. This limit is appropriate for the $\mathrm{Bi}$ - and Tl-based HTSC compounds in a large region of the magnetic field $(H)-T$ plane. In this work, we will use material parameter values appropriate to BSCCO. With these assumptions, one can write the energy of a system of "pancake" vortices residing in the layers as a sum of anisotropic two-body interactions. For straight columnar pins normal to the layers, the pinning potential is the same in every layer. It is then possible to write the free energy as a functional of the time averaged areal vortex density $\rho(\mathbf{r})$ :

$$
F[\rho]-F_{0}=F_{R Y}[\rho]+F_{p}[\rho] .
$$

where $F_{0}$ is the free energy corresponding to a uniform vortex liquid of density $\rho_{0}=B / \Phi_{0}(B$ is the magnetic induction and $\Phi_{0}$ the superconducting flux quantum). The first term in the right-hand side of Eq. (2.1) is the free energy functional in the absence of pinning. As explained above we use for this free energy the RY functional, ${ }^{29}$ which is known ${ }^{30,31,33,34}$ to provide a quantitatively accurate description ${ }^{35}$ of the vortexlattice melting transition in the absence of pinning. It is of the form

$$
\begin{aligned}
\beta F_{R Y}[\rho]= & \int d^{2} r\left(\rho(\mathbf{r})\left\{\ln [\rho(\mathbf{r})]-\ln \left(\rho_{0}\right)\right\}-\delta \rho(\mathbf{r})\right) \\
& -\frac{1}{2} \int d^{2} r \int d^{2} r^{\prime} \widetilde{C}\left(\left|\mathbf{r}-\mathbf{r}^{\prime}\right|\right) \delta \rho(\mathbf{r}) \delta \rho\left(\mathbf{r}^{\prime}\right) .
\end{aligned}
$$

Here $\beta$ is the inverse temperature, $\delta \rho(\mathbf{r}) \equiv \rho(\mathbf{r})-\rho_{0}$, and $\widetilde{C}(r)$ is the usual direct pair correlation function, ${ }^{36}$ which may be written as a sum over layers: $\widetilde{C}(r) \equiv \Sigma_{n} C(n, r)$, with $C(n, r)$ (where $n$ is the layer separation and $r$ the in-layer distance) being the corresponding direct pair correlation function of a layered liquid of pancake vortices. The direct correlation function, which is needed as input in our free energy, can be accurately calculated in a number of ways. We will use here the results of the hypernetted chain calculation of Ref. 34. In general, the results in the limit considered depend on the values of the in-plane London penetration length $\lambda(T)$, the interlayer spacing $d$, and a dimensionless coupling parameter $\Gamma$ given by

$$
\Gamma=\beta d \Phi_{0}^{2} / 8 \pi^{2} \lambda^{2}(T)
$$

For BSCCO we will take $d=15 \AA$, and we will assume a standard two-fluid temperature dependence for $\lambda(T)$ with $\lambda(0)=1500 \AA$ and $T_{c}=85 \mathrm{~K}$ (at zero field).

The second term in the right-hand side of Eq. (2.1) represents the pinning and is of the form

$$
F_{p}[\rho]=\int d^{2} r V_{p}(\mathbf{r}) \delta \rho(\mathbf{r}),
$$

where $V_{p}$ is the pinning potential, which can be written as

$$
V_{p}(\mathbf{r})=\sum_{j} V_{0}\left(\left|\mathbf{r}-\mathbf{R}_{j}\right|\right)
$$

with the sum extending over the planar positions of the random pinning centers. We take the potential $V_{0}$ corresponding to a single pinning center to be of the usual truncated parabolic form: ${ }^{37}$

$$
\beta V_{0}(r)=-\alpha \Gamma\left[1-\left(r / r_{0}\right)^{2}\right] \Theta\left(r_{0}-r\right),
$$

where $r_{0}$ is the range. The basic length in the problem, which we will use as our unit of length unless otherwise indicated, is $a_{0}$, defined by the relation $\pi a_{0}^{2} \rho_{0}=1$. We choose $r_{0}$ to be $r_{0}=0.1 a_{0}$ and take the dimensionless constant $\alpha=0.05$, the value for which, as previously shown, ${ }^{31}$ each pinning center traps approximately one vortex in BSCCO, in the temperature range of interest. This range is determined by the following considerations. We will keep the field fixed at $B$ $=0.2 \mathrm{~T}$ and vary the temperature. The melting temperature of the unpinned lattice is then $T_{m}^{0} \simeq 18.4 \mathrm{~K},{ }^{31}$ and we therefore consider the neighborhood of this temperature. Then one has, to a very good approximation, $\Gamma=2650 / T$ (with the temperature in kelvins), while $\lambda \approx \lambda(0)$.

To carry out our numerical calculations, we discretize the density variables on a computational triangular grid of lattice spacing $h$, containing $N^{2}$ sites. We take the spacing $h$ to be $h=a / 16$ in our calculations, where $a=1.998 a_{0}$ is the equilibrium lattice constant of the vortex lattice in the temperature range considered at the indicated field. This value, as pointed out in Ref. 31, is slightly higher than that of a triangular vortex lattice of density $\rho_{0}$, which is $(2 \pi / \sqrt{3})^{1 / 2} a_{0}$. We define at each site $j$ on this computational lattice a variable $\rho_{j}$, with $\rho_{j} \equiv \rho\left(\mathbf{r}_{j}\right) v$, where $v$ is the area of each computational unit cell. Results reported here are for $N=1024$, which, for the chosen value of $h$, corresponds to including $N_{v}=4096$ vortices in the calculation. Preliminary and confirmatory results at $N=512$ (1024 vortices) were also obtained. A number of pinning sites $\left(N_{p}\right)$ are put, at random, on some of the computa- 
tional lattice sites. The results presented here, which correspond to the dilute limit, will be primarily for a pin concentration $c$ of $c=1 / 64$, that is, a number of pins $N_{p}$ $=64$ at $N=1024$ (or $N_{p}=16$ at $N=512$ ). Some preliminary results for $c=1 / 32\left(N_{p}=128\right.$ at $N=1024$, or $N_{p}=32$ at $N$ $=512$ ) will also be discussed briefly. With random pins, the results depend on the particular random pin configuration and averaging over different such configurations is required. The dependence is however not strong: as we shall see, averaging over five to ten configurations is enough to make statistical errors sufficiently small for our purposes.

To perform our studies, we numerically minimize the discretized free energy with respect to the $N^{2}$ discretized density variables $\left\{\rho_{i}\right\}$. To do so, the interaction term on the right-side of Eq. (2.2) must be repeatedly evaluated, and since this term is of a convolution form, this is most efficiently done in momentum space, through the use of efficient fast Fourier transform (FFT) routines. This avoids having to evaluate this term as a double sum, which would be computationally much more cumbersome than performing the direct and inverse FFT's. In performing the minimization, one must keep in mind that the variables $\left\{\rho_{i}\right\}$ must be non-negative. This precludes the use of many efficient minimization algorithms. We use a procedure ${ }^{38}$ that ensures that this constraint is satisfied. Numerical minimization is performed starting with an appropriate initial condition for the density variables. As explained in Sec. III below, different local minima may be found, at the same field and temperature, depending on the initial conditions. These minima correspond to different phases. The minimization procedure yields not only the value of the free energy at each minimum but also the detailed vortex density configuration at the minimum found, i.e., the values of the set $\left\{\rho_{i}\right\}$ at the minimum. It is then straightforward to analyze the actual density configuration in several ways. It is also possible to evaluate any desired density correlations. The nature of the phase corresponding to each local minimum of the free energy can be inferred from such analysis. The (mean-field) phase diagram is then obtained from a comparison of the free energies of the different minima as the temperature $T$ is varied. The results of carrying out this program are discussed below.

\section{RESULTS}

\section{A. Free energy minima}

As explained above, minima of the free energy are found by starting the minimization process with appropriate initial conditions. We use three different kinds of initial conditions. The first kind is a uniform density $\left(\rho_{i}=\rho_{0} v\right.$ for all $\left.i\right)$, corresponding to a completely disordered liquid state. This is typically used to obtain, as we shall see, liquidlike states at temperatures near the equilibrium melting temperature of the pure vortex lattice, which is ${ }^{30,31} T_{m}^{0} \simeq 18.4 \mathrm{~K}$ for the value of $B$ considered here. The second kind corresponds to a crystalline initial state, with values of $\left\{\rho_{i}\right\}$ as obtained in Ref. 31 for the pure vortex lattice. In our system with a spatially varying random pinning potential, the pinning energy of such a crystalline density configuration depends on the choice of the computational lattice sites at which the periodic local peaks of the density are located. We take, among all possible choices equivalent by symmetry operations in the computational lattice, the one for which the pinning energy is lowest for the specific pinning configuration under consideration. These initial conditions are used to obtain ordered states at lower temperatures. Finally, states originally obtained by either of these two procedures can be slowly warmed up or cooled down: in this case the initial conditions are simply the final state obtained at the previous temperature. As one warms up or cools down a state, its nature in general changes: liquid configurations may become unstable upon cooling and ordered configurations upon warming.

Whichever initial conditions one uses, a local minimum of the free energy is eventually found. At a given temperature, in general several local minima with different density configurations, characterized by the values of the set of $\left\{\rho_{i}\right\}$ variables, are obtained. The minimum with the lowest free energy represents, at a given $T$, the true equilibrium state. It is obviously necessary to develop systematic procedures to identify the structure of each of these diverse minima. Since we have access to the full set of density variables at the computational lattice sites, we have at our disposal a variety of methods to achieve that goal. The first is to calculate the density correlations, e.g. the structure factor $S(\mathbf{k})$ :

$$
S(\mathbf{k})=|\rho(\mathbf{k})|^{2} / N_{v}
$$

where $\rho(\mathbf{k})$ is the discrete Fourier transform of the set $\left\{\rho_{i}\right\}$. Equivalently, one can consider the discretized Fourier transform of $S(\mathbf{k})$, which is the two-point spatial correlation function $g(\mathbf{r})$ of the time-averaged local density. We will present here results for $S(\mathbf{k})$, considered as a function of the vector $\mathbf{k}$, and for the angularly averaged spatial density correlation function, $g(r)$. Since the variables $\rho_{i}$ as defined above are dimensionless, $S(\mathbf{k})$ and $g(r)$ are also dimensionless.

It is also very useful to consider the spatial structure formed by the vortices at low temperatures. For this purpose we have extracted from $\left\{\rho_{i}\right\}$ the local peak densities. We say that the density locally peaks at site $i$ if the value of $\rho_{j}$ at $j$ $=i$ is higher than that at any other computational lattice site within a distance $a / 2$ from $i$, where, as defined above, $a$ is the equilibrium spacing of the unpinned vortex lattice. As expected, we find that at low-temperature solidlike minima where the vortices are strongly localized, the number of local density peaks matches the number of vortices $N_{v}$. The positions of these peaks determine what we will call the "vortex lattice."

Much useful information about the spatial structure of this vortex lattice may be obtained by plotting directly the local density values at the vortex-lattice points. An excellent, and complementary, alternative to help elucidate the degree of vortex-lattice order is to carry out a Voronoi construction: we recall that in the Voronoi construction one determines cells around each lattice point by a Wigner-Seitz procedure. In a perfect crystal, all the resulting Wigner-Seitz cells are identical, while in the general case the cells can have different sizes and shapes. The number of sides of the Wigner-Seitz cell surrounding a lattice point is identified with the number 


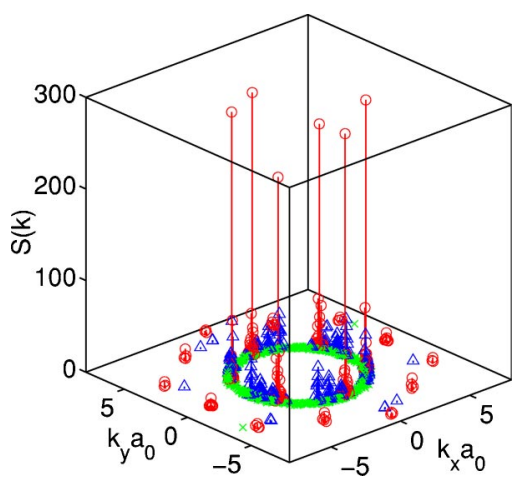

FIG. 1. (Color online) The static structure factor $S(\mathbf{k})$ [see Eq. (3.1)] for three local minima obtained at $T=18.4 \mathrm{~K}$ for the same pin configuration. The (red) circles are for a state identified, from the overall evidence (see text), as a Bragg glass (BrG), the (blue) triangles are for a Bose glass (BoG) state, and the (green) crosses are for an interstitial liquid (IL). The vertical lines are guides to the eye.

of "nearest neighbors" of that lattice point, and the difference between this number and the average (six in our case) marks the position of defects (disclinations). We will make use of such plots below.

Since we will be concerned not only about translational, but also with orientational order, we will also examine the bond-orientational correlation function $g_{6}(r)$ in the vortex lattice defined as

$$
g_{6}(r)=\langle\psi(\mathbf{r}) \psi(0)\rangle,
$$

where the angular brackets denote the overall average over the vortex lattice and the field $\psi(\mathbf{r})$ is given by

$$
\psi(\mathbf{r})=\frac{1}{n_{n}} \sum_{j=1}^{n_{n}} \exp \left[6 i \theta_{j}(\mathbf{r})\right]
$$

with $\theta_{j}(\mathbf{r})$ being the angle that the bond connecting a vortex at $\mathbf{r}$ to its $j$ th neighbor makes with a fixed axis, and $n_{n}$ is the number of neighbors of the vortex at $\mathbf{r}$.

It is also possible, and as we shall see, useful, to define what we will call a "translational correlation function" in the vortex lattice in a way that is quite analogous to the definition of $g_{6}(r)$. This function, which we denote as $g_{G}(r)$, is defined by an equation identical to the right-hand side of Eq. (3.2a), but with the field $\psi$ being defined as

$$
\psi_{G}(\mathbf{r})=\exp (i \mathbf{G} \cdot \mathbf{r})
$$

where $\mathbf{G}$ is a reciprocal-lattice vector of the triangular vortex lattice in the absence of pinning. We will consider here only the case where $\mathbf{G}$ is a shortest nonzero reciprocal-lattice vector and average over the results obtained for the six equivalent G's.

Our identification of the different phases is based on analysis of all this information. We show some of the results in the next few figures. These all correspond to samples with 64 pins and 4096 vortices. First, in Fig. 1, we show the structure factor, as defined in Eq. (3.1). The three sets of results shown there are for local free energy minima at the same temperature, $T=18.4 \mathrm{~K}$, obtained with different initial conditions of the three kinds described above, and the same pin configuration. Other pin configurations yield very similar results (a different example is shown in Fig. 1c of Ref. 32). The green symbols correspond to the free energy minimum obtained by starting with uniform initial conditions. Clearly, the structure factor is completely featureless and liquidlike in this case-the absolute value of $S$ never exceeds 5 . The red circles are for a local minimum obtained with initial conditions corresponding to the best crystalline state, as explained above, for this pin configuration. The structure factor now exhibits typical ordered behavior, highlighted by six sharp Bragg-like peaks that are emphasized by the added vertical lines in the figure. Finally, the blue triangles correspond to a minimum obtained by first "quenching" with uniform initial conditions to a relatively low temperature $(16.8 \mathrm{~K})$ where the liquidlike state is found not to be stable, and then slowly warming, at $0.2 \mathrm{~K}$ temperature intervals, back up to $18.4 \mathrm{~K}$. As one can see, the $S(\mathbf{k})$ for this minimum has an intermediate structure, with several relatively well-defined peaks, more than six in number, whose heights are considerably lower than those of the peaks found for the ordered minimum.

In Fig. 2, results for the angularly averaged density correlation function $g(r)$ are displayed. These results are for minima obtained at the same temperature, and using the same initial condition procedures, as those for the data shown in Fig. 1. However, the results shown here are averages over five different pin configurations. The nearly flat (green) curve in the top panel corresponds to minima obtained from quenches with uniform initial conditions, the (red) curve with the well-defined peaks shown in the bottom panel corresponds to minima obtained with crystalline initial conditions, and the (blue) curve with the intermediate peaks shown in the top panel is for minima obtained by quenching with uniform initial conditions to a low temperature and subsequent slow warming (very similar results can alternatively be obtained by slowly cooling a high-temperature liquidlike minimum to a temperature where it is unstable). One can see then that the results for $g(r)$ are fully consistent with those found in Fig. 1: the minimum obtained from uniform initial conditions is fully disordered, while that obtained from crystalline initial conditions exhibits a large degree of crystalline order. For the third kind of minima, we find some degree of intermediate range order.

We next examine the structure of the minima in terms of the "vortex lattice" found by the procedure explained above. Sample results are shown in Fig. 3, all for the same pin configuration, at the temperature $T=18.2 \mathrm{~K}$. Results for three minima, obtained from the same procedures and initial conditions as the three minima in the previous two figures, are shown. The positions of the local density peaks and the values of the density at these peaks are displayed through a symbol and color coding scheme described in the caption of the figure. Blank regions denote areas where no local peak was found using the algorithm described above: such areas are mostly found only in the most disordered case (uniform initial conditions). We see that the results are quite consistent with those obtained from the previous correlation functions: quenching with the appropriate crystalline initial conditions leads to a fairly well-ordered (but not perfectly ordered) lat- 

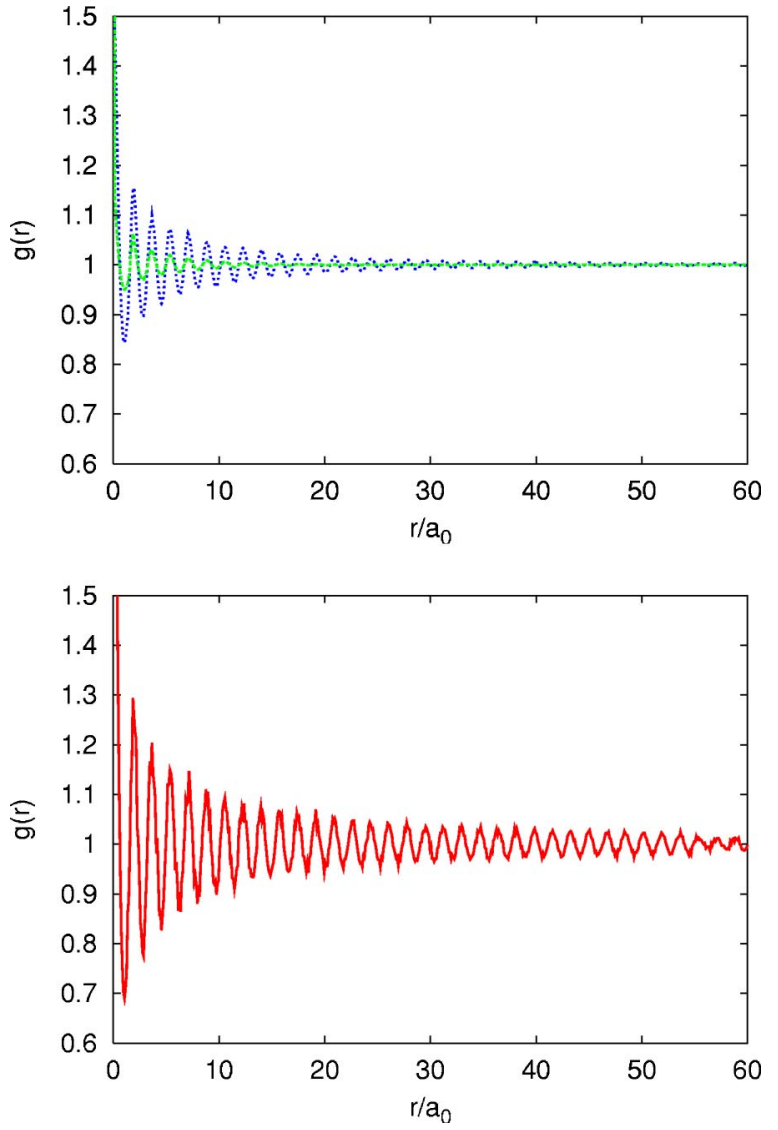

FIG. 2. (Color online) The angularly averaged density correlation function $g(r)$ plotted at the same temperature as in Fig. 1 as a function of dimensionless distance. The results are averaged over five different pin configurations. The results for the BoG and IL minima are shown in the top panel. The (blue) dotted line plot with higher peaks corresponds to BoG minima and the (green) light gray plot with fewer and lower peaks to IL minima. The bottom panel displays the results for the BrG minima. The vertical and horizontal scales are the same in the two panels and the color scheme is the same as that in Fig. 1.

tice, while from uniform initial conditions, one obtains a rather disordered, liquidlike structure where the density is nearly uniform and close to $\rho_{0}$, except near the pinning centers at each of which one vortex is trapped. An intermediate result, on the whole more solid than liquidlike, but definitely disordered, is obtained through the slow warming or cooling scheme.

To better gauge the degree of disorder present in each case, we construct, in Fig. 4, the corresponding Voronoi plots. To display the behavior in a more obvious way, the temperature in these plots is lower by $0.2 \mathrm{~K}$ than that of the plots in Fig. 3. As mentioned above, sites with number of nearest neighbors $\left(n_{n}\right)$ different from 6 represent the locations of disclinations. In the solidlike minima (top two panels of Fig. 4), nearly all the sites with $n_{n} \neq 6$ have $n_{n}=5$ or $n_{n}$ $=7$ (the disclinations have unit "charge"). Also, sixfold- and sevenfold-coordinated sites, indicated, respectively, by (red) triangles and (green) solid circles in the plots, always occur in nearest-neighbor pairs. Such pairs correspond to dislocations. In the first panel, which displays the results for the
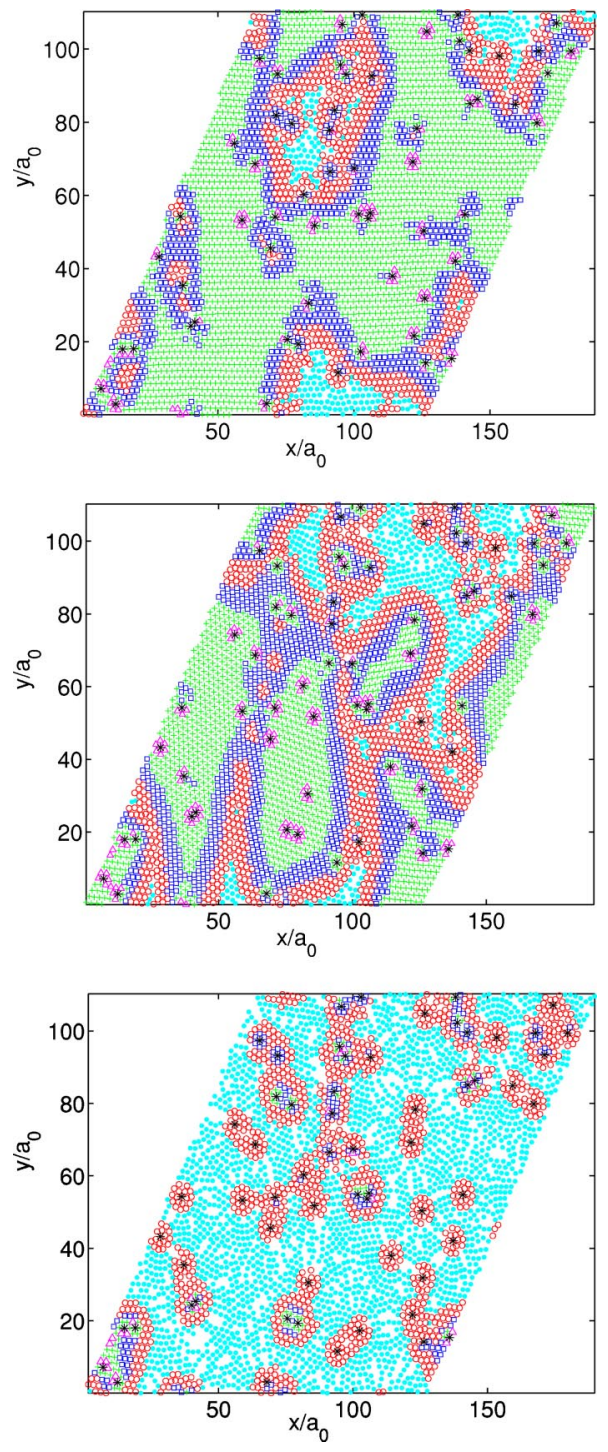

FIG. 3. (Color online) Local peak densities at three different local minima of the free energy. The positions of the local density peaks and the values of the density at these peaks are displayed according to the following scheme: (cyan) solid circles, peaks with $\rho_{\text {peak }} / \rho_{0}<1.5$; (red) circles, $1.5 \leqslant \rho_{\text {peak }} / \rho_{0}<3.5$; (blue) squares, $3.5 \leqslant \rho_{\text {peak }} / \rho_{0}<5.5$; (green) plus signs, $5.5 \leqslant \rho_{\text {peak }} / \rho_{0}<7.5$; and (purple) triangles, $7.5 \leqslant \rho_{\text {peak }} / \rho_{0}<9.5$. Blank areas, found mainly in the bottom panel, correspond to regions where no local peaks are found. The temperature is $18.2 \mathrm{~K}$ in all panels, which all correspond to the same pin configuration as that for the results in Fig. 1. Pin locations are indicated by (black) asterisks. From top to bottom, the results displayed correspond, respectively, to ordered (identified as $\mathrm{BrG})$, intermediate (BoG), and disordered (IL) minima.

most ordered state obtained from crystalline initial configurations, we see that the number of dislocations, while nonzero, is quite small and that they form fairly isolated small clusters, each of which has zero net Burgers vector. These defect clusters occur near the pinning centers and they represent the local disruption of crystalline order due to the pinning. In the second panel, which shows the results for the minimum with intermediate order, the dislocations are organized to form well-defined grain boundaries that separate 

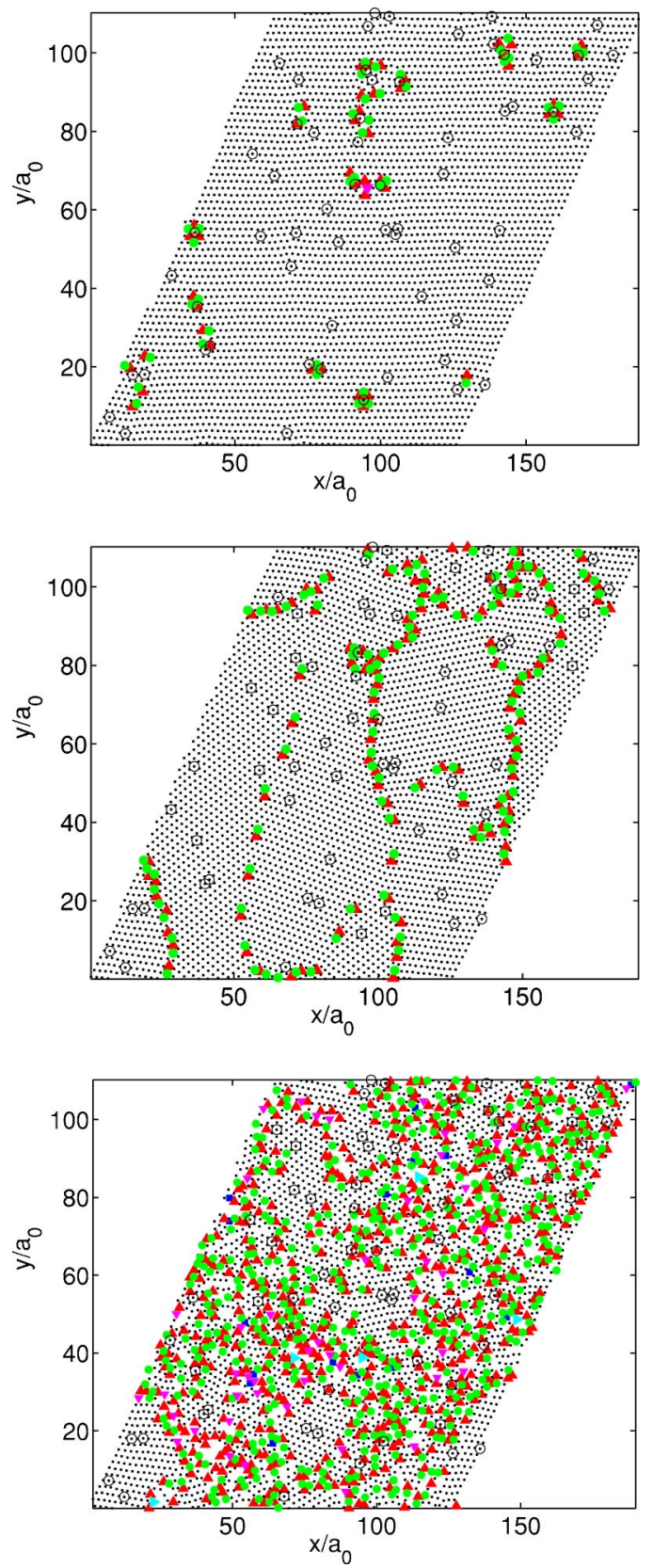

FIG. 4. (Color online) Voronoi plots for the three cases for which density plots are shown in Fig. 3, except that the temperature is now $T=18.0 \mathrm{~K}$. The (black) dots denote lattice sites with six neighbors, the (red) triangles denote fivefold-coordinated sites, and the (green) solid circles, sevenfold-coordinated sites. Rarely occurring fourfold- and eightfold-coordinated sites are indicated by (blue) squares and (purple) inverted triangles, respectively. Sites surrounded by black circles denote locations of pinning centers. See text for explanations.

crystallites with different orientations. As a result of this polycrystalline structure, both translational and bondorientational correlations are short-range. Performing the same construction for other pin configurations (results for a different pin configuration are shown in Fig. 1b of Ref. 32), one finds that the crystallite arrangement depends on the pin configuration, as one would expect. The grain boundaries

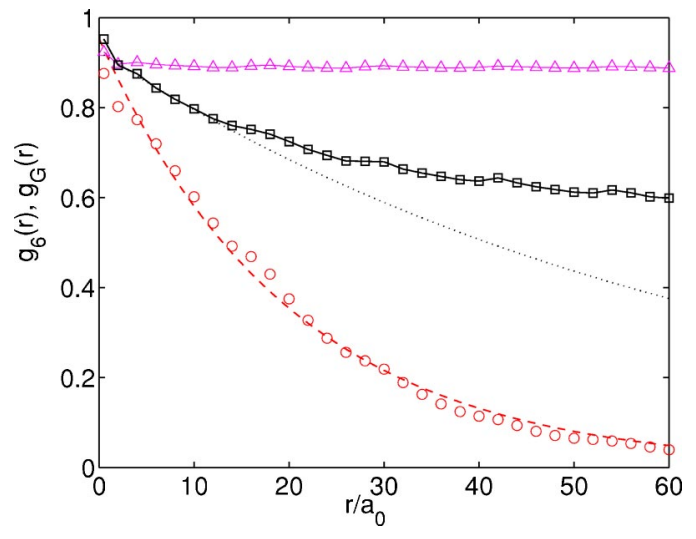

FIG. 5. (Color online) Bond-orientational correlation function $g_{6}(r)$ as defined in Eq. (3.2a), and "translational" correlation function, $g_{G}(r)$ [see Eq. (3.3)]. These are results averaged over five pin configurations, at $T=17.6 \mathrm{~K}$. The (purple) triangles and (red) circles denote $g_{6}(r)$ for ordered $(\mathrm{BrG})$ and polycrystalline $(\mathrm{BoG})$ minima (liquidlike minima are not stable at this lower $T$ ), respectively. The black squares are $g_{G}(r)$ for the $\mathrm{BrG}$ minima. The dashed line is an exponential fit to $g_{6}(r)$ for the BoG minima. The dotted line is a similar exponential fit to $g_{G}(r)$ for the BrG minima at small distances, showing that the decay is slower than exponential for large values of $r$. The solid lines connecting symbols are guides to the eye.

tend to lie away from pinning centers, which makes sense physically. Finally, in the last panel, which shows the results for the minimum obtained from uniform initial conditions, the high degree of disorder is evident. The Voronoi construction shown in this panel is not very meaningful because our method of obtaining the vortex positions is not reliable for liquidlike minima. The identification of the position of a vortex with that of a local peak of the density field is justified only when the peak is sharp. This is the case in the solidlike minima, but not so in the liquidlike minima with low peak densities. We have shown here the results for the liquidlike minimum only for the purpose of illustrating the difference between the structure of this minimum and those of the more ordered ones. For this minimum, we find a very large number of defects, and it is difficult to determine whether disclinations of opposite "charge" always pair up to form dislocations. Inspection of the defect distribution suggests the presence of free disclinations, but not conclusively. The total number of local peaks of the density field in liquidlike minima turns out to be substantially smaller than the expected number of vortices. This problem is not present at the solidlike minima where nearly all the vortices are strongly localized.

Next, we show in Fig. 5 examples of the bondorientational and "translational" correlation functions, $g_{6}(r)$ and $g_{G}(r)$ as defined in Eqs. (3.2a), (3.2b), and (3.3), respectively. All results shown in this figure are averages over five pin configurations at temperature $T=17.6 \mathrm{~K}$. At this temperature the liquid is unstable, and in any case all liquid correlation functions are featureless, so that case is not shown. The (purple) triangles correspond to ordered minima obtained from quenching with crystalline initial conditions to $T$ $=16.8 \mathrm{~K}$ and subsequent slow warming, and represent the 
bond-orientational correlation, $g_{6}(r)$. This function appears to saturate at a fairly large value as $r$ increases, indicating the presence of long-range bond-orientational order. The (red) open circles display $g_{6}(r)$ for the polycrystalline state with intermediate order, obtained through the procedures described above. This function decays exponentially with distance, as shown by the exponential fit (dashed line), over a distance of few tens in units of $a_{0}$. Finally, the (black) open squares show the results for the "translational" correlation function $g_{G}(r)$ for the ordered minima. The dotted line is an exponential fit to this translational correlation function for $r / a_{0}<10$, showing that the decay at longer distances is clearly slower than exponential (possibly a power law).

From the overall examination of data such as those shown in these figures, we can reach the following conclusions: quenching with uniform initial conditions to temperatures above or slightly below $T_{m}^{0}$ leads to very disordered states, with very-short-range correlations and no structure, except for vortices at and near the pinning centers. These minima become unstable upon slow cooling: one then obtains minima of the third kind, discussed below. They clearly must be identified with the liquid phase, specifically the IL phase discussed in the Introduction. The second kind of minima is obtained by quenching with crystalline initial conditions to any temperature below, or slightly above, $T_{m}^{0}$. These minima can then be cooled, without losing their character, but they melt into the IL upon sufficient warming. The states thus obtained are obviously nearly crystalline, with the reservation that the order is not truly long range, but has a slow decay. This is most evident in the results for $g_{G}(r)$ seen in Fig. 5. The bond-orientational order is nearly perfect. Defects are limited to isolated clusters. This state must therefore be identified as a BrG. Finally, we have the states obtained as described above, by slow cooling of the IL state, which can also be obtained by quenching with uniform initial conditions to a temperature below the IL state stability limit. Either procedure yields, for any pin configuration, states that at a given $T$ differ only slightly in free energy or density configuration. The states do differ somewhat more, however, for different pin configurations. The Voronoi construction conclusively shows (see Fig. 4) that these states are polycrystalline. The grain boundaries are formed by dislocation chains. For any pin configuration, these grain boundaries survive thermal cycling across $T_{m}^{0}$, but if sufficiently warmed up, these minima melt, the melting beginning locally at the grain boundaries (see Sec. III B below). The bond-orientational correlation function for these minima has an intermediate range. This, and also the observation that these states are not unique (as mentioned above, slightly different states are reached depending on the cooling or quenching protocol), clearly indicate a glassy state. We therefore identify it with the BoG, which has been experimentally ${ }^{18,19}$ and numerically ${ }^{25}$ shown to be polycrystalline.

\section{B. Properties of the condensed phases}

In this section, we describe in detail how the properties of the low-temperature "solidlike" condensed phases represented by the $\mathrm{BrG}$ and $\mathrm{BoG}$ types of minima defined in the preceding section vary as the temperature is changed. The high-temperature liquid phase represented by the IL-type minima is not very interesting from the point of view of its temperature dependence: in the temperature range we have considered, this phase exhibits a liquidlike (nearly uniform) density distribution except in the immediate vicinity of the columnar pins, at each of which a vortex is trapped. These trapped vortices would eventually get delocalized at higher temperatures. This happens beyond the upper limit of the temperature range $(16.8 \mathrm{~K} \leqslant T \leqslant 18.8 \mathrm{~K})$ we have considered here.

To study how the properties of a local minimum of the free energy change as the temperature is varied, we have "followed" minima as the temperature changes: for example, starting with a minimum obtained by quenching to the lowest temperature of $16.8 \mathrm{~K}$, we "follow" that minimum to higher temperatures by increasing the temperature in small steps (usually taken to be $0.2 \mathrm{~K}$ ) and finding a new minimum at the higher temperature by running the minimization routine with the configuration at the minimum obtained at the previous temperature as the initial state. This procedure leads to a new minimum of the same type as long as the minimum remains locally stable-if the temperature is increased to values substantially higher than $T_{m}^{0}$, where the $\mathrm{BrG}$ and $\mathrm{BoG}$ minima become unstable, this procedure leads then to the IL minima. We have also carried out "cooling" runs where the temperature was decreased in small steps, starting from a minimum obtained at a relatively high temperature. When the IL minima become unstable, BoG states are obtained. As long as the minimum under consideration did not become unstable in the range of temperatures considered in the runs, we did not find any substantial difference between the results obtained in the "heating" and "cooling" runs.

To characterize the density distribution at a minimum, we looked at "global" quantities such as the structure factor $S(\mathbf{k})$, an example of which is shown above in Fig. 1. The $T$-dependent information contained in $S(\mathbf{k})$ is, however, difficult to display in plots. We therefore introduce a closely related "order parameter" $m$ defined as

$$
m \equiv \sqrt{\left(S_{\max } / N_{v}\right)},
$$

where $S_{\max }$ is the largest value of the structure factor $S(\mathbf{k})$ averaged over the six $\mathbf{k}$ vectors related by lattice symmetry to each $\mathbf{k}$. By definition, the order parameter $m$ is equal to unity in a state with perfect crystalline order (triangular lattice with $\delta$-function peaks). Since no long-range crystalline order is expected or found in either one of the $\mathrm{BrG}$ and $\mathrm{BoG}$ phases, the value of $m$ should go to zero at all temperatures in the thermodynamic limit. However, the infinite size limit is reached very slowly in the glassy phases, and for the finite-size systems considered here, this quantity provides a convenient measure of the degree of local order present at the minima.

We show in Fig. 6 the results for the order parameter $m$, averaged over five different pin configurations, for samples with 4096 vortices and 64 pins. The results for the BrG, $\mathrm{BoG}$, and IL minima are shown by (red) plus signs, (green) crosses, and (blue) asterisks, respectively. Only two data 


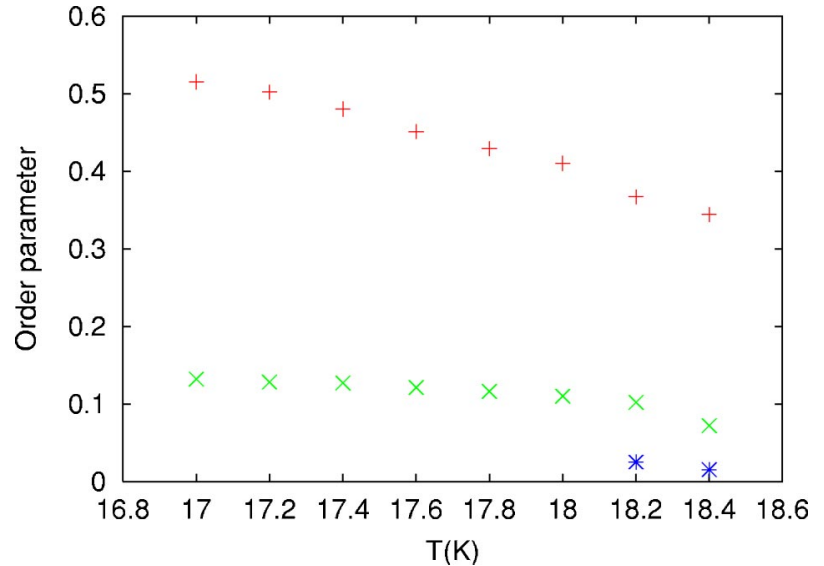

FIG. 6. (Color online) Temperature dependence of the order parameter $m$ as defined in Eq. (3.4) for three kinds of minima, averaged over five samples, with $N_{v}=4096$ and $N_{p}=64$. Results for the $\mathrm{BrG}$, BoG, and IL minima are shown by (red) plus signs, (green) crosses, and (blue) asterisks, respectively.

points are shown for the IL minimum because it becomes unstable at lower temperatures. For all three kinds of minima, the value of $m$ decreases with increasing $T$, as expected. The rate of change is largest for the BrG minima, and smallest for the IL minima. However, the differences among the three kinds of minima in the degree of local order remain quite clear at all the temperatures considered. We have also obtained BrG-type minima for several $(\sim 10)$ samples with 1024 vortices and 16 pins. The values of $m$ obtained from an average over these smaller samples are found to be only about 6\% larger than those obtained for the 4096-vortex samples at the corresponding temperature. This result implies that the translational correlation function at the $\mathrm{BrG}$ minima falls off very slowly with distance. This is consistent with our interpretation of these minima as representing the $\mathrm{BrG}$ phase.

We also examined the temperature dependence of the detailed density distribution at the minima (obtained from "peak-density" plots similar to those shown in Fig. 3) and their defect structure obtained from Voronoi plots such as those in Fig. 4. Examination of the peak-density plots reveals more information about how the density distribution at the minima changes with temperature. In Fig. 7, we have shown peak-density plots at two temperatures $(17.4 \mathrm{~K}$ in the top panel and $17.8 \mathrm{~K}$ in the bottom panel) for the BoG minimum of the same sample for which a similar plot at $18.2 \mathrm{~K}$ is shown in the middle panel of Fig. 3. The symbols and color scheme used in these plots are the same as those in Fig. 3. It is clear from these plots that the typical values of the local peak densities decrease (the vortices become less localized) as the temperature increases. The largest values of the local peak density occur near the pinning centers and the smallest values appear near the grain boundaries that separate different crystalline regions. This is physically reasonable-the disruption of local crystalline order near the grain boundaries should make the vortices in such regions more delocalized. At the relatively low temperature of $17.4 \mathrm{~K}$, there are very few local density peaks where the peak density is lower than $3.5 \rho_{0}$ [such peaks are indicated by (red) circles in the plot].
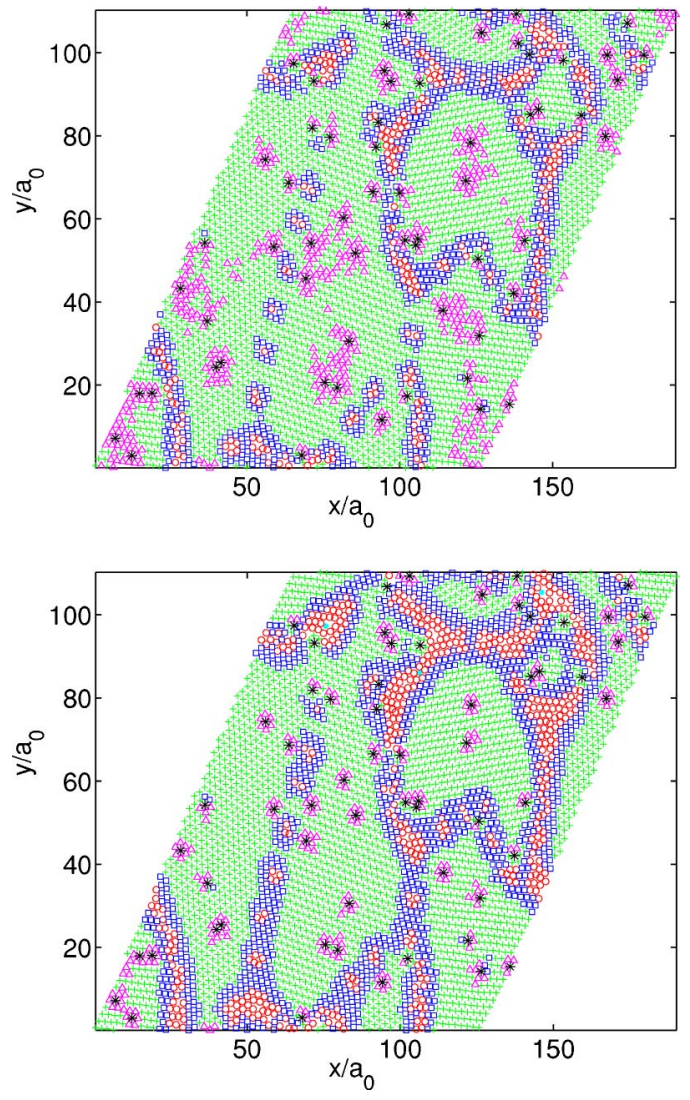

FIG. 7. (Color online) Peak density plots for the BoG minimum of a sample with $N_{v}=4096$ and $N_{p}=64$ (the sample is the same as the one for which results are shown in Figs. 3 and 4) at two temperatures: $T=17.4 \mathrm{~K}$ (top panel) and $T=17.8 \mathrm{~K}$ (bottom panel). The symbols and color scheme used here are the same as those in Fig. 3.

As the temperature is increased to $17.8 \mathrm{~K}$, the number of such peaks increases, and this trend continues as $T$ is increased further, as can be seen in the middle panel of Fig. 3 where the results for $T=18.2 \mathrm{~K}$ are shown. It is also clear from these plots that the spatial regions where the low peak densities occur remain roughly unchanged as the temperature increases-as noted above, these regions are strongly correlated with the locations of the grain boundaries. These observations indicate that the vortices in the interior of the crystalline grains remain in a "solid" state as the temperature is increased to a value close to $T_{m}^{0}$, while those in the neighborhood of the grain boundaries begin to "melt" (get delocalized) at a lower temperature. This "inhomogeneity" of the melting process will be discussed in more detain in Sec. III D.

We also studied the temperature dependence of the number of topological defects present at the minima. This number increases slowly with increasing $T$, and then exhibits a sudden jump as liquidlike regions (characterized by low values of the local peak densities) appear near the grain boundaries at temperatures close to $T_{m}^{0}$. As noted in Sec. III A above, our method of obtaining the defect structure using the Voronoi construction becomes somewhat less reliable when liquidlike regions begin to appear. Due to this difficulty in obtaining reliable results for the defect structure and statistics 
at relatively high temperatures, we have not carried out a quantitative analysis of the dependence of these quantities on the temperature.

The gradual decrease in the values of the local peak densities with increasing temperature is also found in the $\mathrm{BrG}$ minima, but on the average the vortices remain more strongly localized at the $\mathrm{BrG}$ minima than at the corresponding $\mathrm{BoG}$ ones. The strong correlation between low values of the local peak density and the location of topological defects is found at the $\mathrm{BrG}$ minima too. The main difference between $\mathrm{BrG}$ and $\mathrm{BoG}$ minima is that the defects are more randomly distributed at the $\mathrm{BrG}$ minima: they do not line up along grain boundaries as they do at the BoG minima. For this reason, the regions of low peak density appear at fairly random locations at the $\mathrm{BrG}$ minima. The total number of topological defects at the $\mathrm{BrG}$ minima remains smaller than that at corresponding BoG minima at all temperatures. As the temperature approaches $T_{m}^{0}$, liquidlike regions appear in parts of the sample where the defect density is large. This is illustrated in the plots in the top panels of Figs. 3 and 4.

Thus, our investigation of the temperature dependence of the structure of the BrG and BoG minima confirms that these minima retain their topological structure as $T$ is increased toward $T_{m}^{0}$ and even beyond (provided they remain stable). The degree of localization of the vortices decreases with increasing $T$ and liquidlike regions with nearly delocalized vortices begin to appear at temperatures close to $T_{m}^{0}$. However, even at such temperatures, the BrG and BoG type minima are clearly distinguishable from one another and from the IL-type minima, as illustrated in Figs 3, 4, and 6.

\section{Two-step melting transition}

As discussed in the previous subsections, we find three kinds of coexisting, locally stable minima of the free energy at temperatures close to $T_{m}^{0}$. Two kinds ( $\mathrm{BrG}$ and $\left.\mathrm{BoG}\right)$ of local minima are found at temperatures substantially lower than $T_{m}^{0}$, and only the IL minimum is stable if $T$ is much higher than $T_{m}^{0}$. In our mean-field description, the thermodynamically stable phase at a particular temperature corresponds to the minimum with the lowest free energy. Therefore, crossings of the free energies of different kinds of minima correspond to first-order phase transitions in our description. The IL minima are expected to have the lowest free energy at high temperatures and the solidlike $\mathrm{BrG}$ or BoG minima should represent the globally stable phase at low temperatures. To determine how this "freezing" transition takes place, it is necessary to examine the dependence of the free energies of these different kinds of minima on the temperature $T$.

In Fig. 8, we have shown the results for the free energies of different minima of a sample with $N_{v}=4096$ and $N_{p}=64$. This sample is the same as the one for which results are shown in Figs. 1, 3, 4, and 7. A similar plot, showing the same general behavior for a different sample, may be found in Ref. 32. The BrG minimum clearly has the lowest free energy at low temperatures (while we have shown data for $T \geqslant 17.8 \mathrm{~K}$, we have checked that this remains true at lower temperatures), while the IL minimum is the one with the

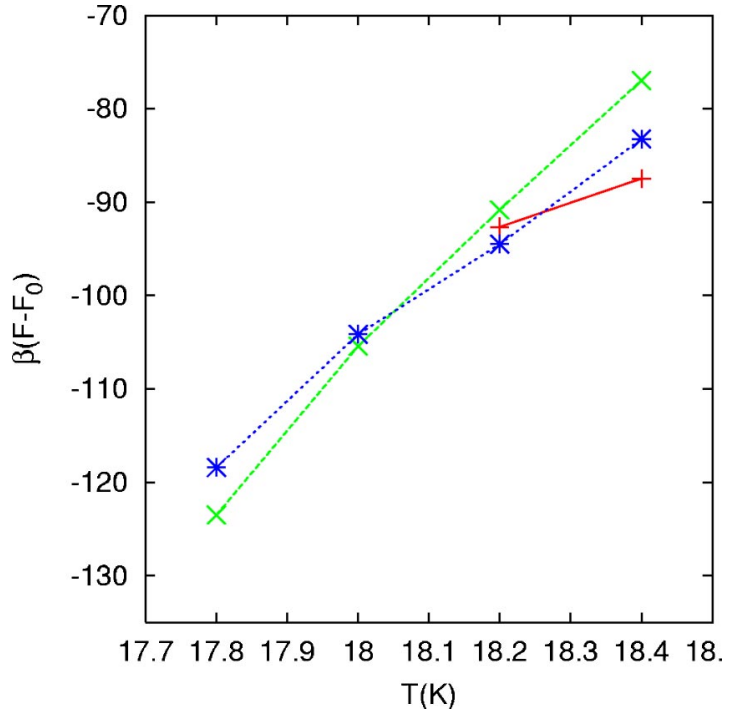

FIG. 8. (Color online) Temperature dependence of the dimensionless free energies of different local minima of a sample with $N_{v}=4096$ and $N_{p}=64$ (the sample is the same as the one for which results are shown in Figs. 1, 3, 4, and 7). The data for the $\mathrm{BrG}$, BoG, and IL minima are shown by the (green) crosses and dashed line, (blue) asterisks and dotted line, and the (red) plus signs and solid line, respectively. The lines are guides to the eye.

lowest free energy at high temperatures. The interesting feature that emerges from data as that shown in this figure is that the BoG minimum has the lowest free energy in an intermediate temperature range of small width (between 18.0 and $18.3 \mathrm{~K}$ for this sample), indicating that the melting of the low-temperature solid phase to the high-temperature IL phase occurs in two distinct steps: the BrG phase that is the thermodynamically stable one at low temperatures undergoes a first-order transition into a BoG phase as the temperature is increased, and then this BoG phase melts into the IL phase via a second first-order transition at a slightly higher temperature. This two-step melting behavior is one of our main results.

The same qualitative behavior, indicating the occurrence of two separate first-order transitions, is found in all the 4096-vortex samples we have studied at pin concentration $c=1 / 64\left(N_{p}=64\right)$. The average value of the temperature interval in which the BoG phase has the lowest free energy is about $0.42 \mathrm{~K}$. This width exhibits fairly large sample-tosample variations, ranging between about $0.1 \mathrm{~K}$ in one sample and a maximum of $1.2 \mathrm{~K}$. As the system is cooled from the high-temperature liquid phase, it undergoes a firstorder transition into a polycrystalline $\mathrm{BoG}$ phase. Such a transition has been observed ${ }^{19}$ in experiments on BSCCO samples with a small concentration of columnar pins. The value of the upper (BoG to IL) transition lies between 18.2 and $18.3 \mathrm{~K}$ in all the 4096-pin samples we have studied. These values are quite close to the first-order melting temperature $T_{m}^{0}$ of the same system in the absence of pins. ${ }^{31}$ Our results, thus, are consistent with the experimental observation $^{17,18}$ of a weak dependence of the freezing temperature of the vortex liquid on the pin concentration $c$ for small values of $c$. In addition, our work predicts a second 
first-order transition to a more ordered $\mathrm{BrG}$ phase at a slightly lower temperature.

For smaller samples at the same $c=1 / 64$ concentration ( $N_{v}=1024$ with $N_{p}=16$ ) we did not always find the BoG minima: the minimization procedure that led to the $\mathrm{BoG}$ minima in the samples with $N_{v}=4096$ often converged to minima of the BrG type in the smaller samples. This is because the typical size of the crystalline grains at the BoG minima at this pin concentration is of the order of the sample size for 1024-vortex samples. This can be seen by comparing the middle panel of Fig. 9, where we have shown the Voronoi plot for the BoG minimum obtained for a $N_{v}=1024$ sample, with the Voronoi plot in the middle panel of Fig. 4, which is for the same $T$ and $c$, but a larger sample with 4096 vortices. One can plainly see that the domain size is comparable to the system size of the smaller samples. However, for the 1024vortex samples where we found BoG-type minima, the behavior of the free energies was found to be very similar to that shown in Fig. 8. An example of such behavior is shown in the bottom panel of Fig. 9. The two-step melting transition found in the larger systems is found here also, indicating that this is the generic behavior. We have also carried out preliminary studies of the phase diagram for a slightly higher value of the pin concentration, $c=1 / 32$. We find the same qualitative behavior, namely the occurrence of two-step melting, with a larger difference between the two transition temperatures. We therefore conclude that the two-step melting transition we have found is a characteristic feature of our model of layered superconductors with a small concentration of columnar pins.

Since a first-order transition is not characterized by power-law behaviors of thermodynamic quantities with universal exponents, the usual finite-size scaling analysis of numerical data for continuous transitions does not apply to our work. However, we have examined the dependence of the magnitudes of various discontinuous changes at the transitions on sample size. From the results for the free energy as a function of the temperature, we have calculated the entropy jump per vortex at the first-order transitions. The entropy change at the $\mathrm{BrG}-\mathrm{BoG}$ (lower temperature) transition is found to be $\simeq 0.1 k_{B}$ per vortex ( $k_{B}$ is the Boltzmann constant), and the corresponding change at the BoG-IL (higher temperature) transition has a slightly higher value, $\simeq 0.15 k_{B}$ per vortex. The sum of these two entropy jumps is slightly smaller than the entropy change $\left(\simeq 0.29 k_{B}\right.$ per vortex $)$ at the single first-order melting transition found ${ }^{31}$ in the same system in the absence of pinning. All these results are physically reasonable. The difference between the degrees of order in the $\mathrm{BrG}$ and $\mathrm{BoG}$ minima is smaller than that between the BoG and IL minima, suggesting that the entropy jump at the lower transition should be smaller than that at the upper one. Also, the low-temperature BoG phase of the system in the presence of pinning is less ordered than the crystalline phase of the pure system, and the IL phase is slightly more ordered than the vortex liquid in the system without pinning (this is due to the local order of the vortices near the pinning centers which persists in the IL phase). Therefore, the net change of entropy in going from the BoG phase to the IL phase should be smaller than the entropy jump at the melting transition in the pure system. A comparison of our results for two sample
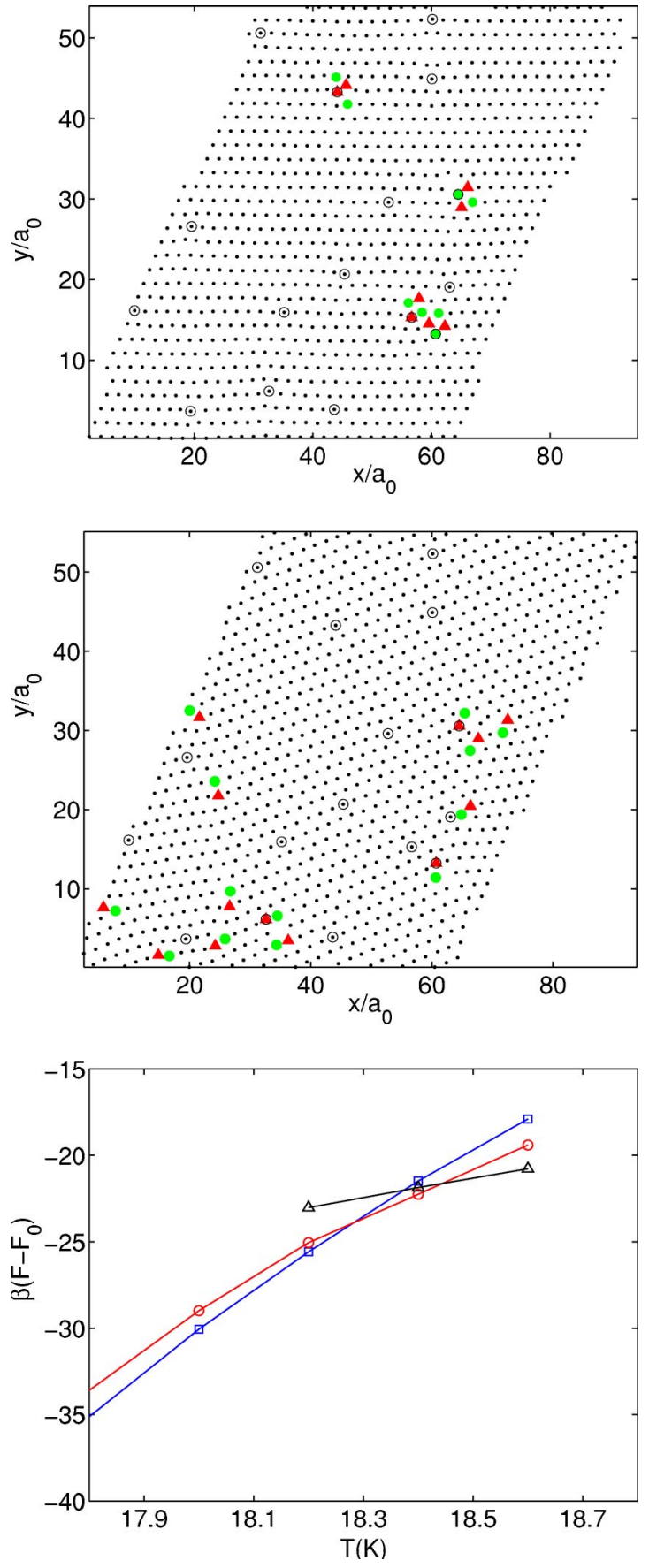

FIG. 9. (Color online) Results for a sample with $N_{v}=1024$ and $N_{p}=16$. Voronoi plots for the $\mathrm{BrG}$ and $\mathrm{BoG}$ minima at $18.0 \mathrm{~K}$ are shown in the top and middle panels, respectively. The symbols and color scheme used in these plots are the same as those in Fig. 4. The bottom panel shows the temperature dependence of the free energies of the BrG (blue line and squares), BoG (red line and circles), and IL (black line and triangles) minima, respectively. The lines are guides to the eye.

sizes $\left(N_{v}=4096\right.$ and 1024) does not show any appreciable dependence of the entropy changes on the sample size. We therefore conclude that there is no indication of any significant change in our phase diagram as the sample size is increased. 
Since our mean-field treatment ignores the effects of fluctuations, it is important to address the question of whether one or both of the transitions found would become continuous if fluctuations were included. However, there are very few examples of such fluctuation-driven continuous transitions in three dimensions. In our calculations, the effects of the electromagnetic interaction among vortices on different layers are included. So, we expect our results regarding the nature of the transition to remain valid if fluctuations were included. This conclusion is supported by the result that our density functional theory provides a quantitatively correct account $^{35}$ of the first-order melting of the vortex lattice in the pure system: it can be expected that the agreement between theory and experiment for the pure limit will carry on to the dilute pinning case we study here. Also, the transition in the presence of a small concentration of columnar defects is known to be first-order both experimentally ${ }^{17-19}$ and from simulations. ${ }^{27}$

The heights of free-energy barriers between minima cannot be calculated from our procedures. However, a very rough estimate of the barrier heights can be made through a physical argument: as already mentioned, the minima we find can become unstable upon rather small changes of $T$. The corresponding changes in the dimensionless free energy are seen (e.g., in Fig. 6) to be of the order of the free energy differences between minima in the temperature range considered. It is very hard to imagine how a local minimum could disappear in this way unless the barrier heights themselves were also of the same order.

The appearance of a sliver of the BoG phase in our phase diagram may be qualitatively understood as arising from a competition between the elastic and pinning parts of the free energy. The elastic (free) energy plays a dominant role in the $\mathrm{BrG}$ minima: the effect of the randomly located pinning centers is accommodated in this structure by small displacements of the vortices from their ideal lattice positions towards the nearest pinning center. Where the occupation of a pinning center by a vortex would require a large displacement of the vortex, the pinning center is not fully occupied. For this reason, the number of pinning centers occupied by vortices at a $\mathrm{BrG}$ minimum is always slightly lower than the total number of pinning centers. At the corresponding BoG minima, on the other hand, the pinning centers are always occupied (except in very rare cases where two pinning centers are located very close to each other). Since the BoG minima are obtained from liquid-like initial conditions, the starting point has fully occupied pinning centers, and vortices localized in small crystalline patches in their immediate vicinity (see, for example, the bottom panel of Fig. 3 which shows such crystalline patches surrounding the pinning centers). The crystalline orientation of vortices around a vortexbinding pin site depends on the local arrangement of the pins. ${ }^{37}$ This orientation is, in general, different in different regions of the sample. These crystalline patches grow as the temperature is reduced, and eventually meet one another at grain boundaries to form a polycrystalline BoG minimum. It is clear that the pinning centers are better accommodated in this structure than in the corresponding BrG structure. On the other hand, the creation of grain boundaries costs elastic energy. Thus, the BoG minima are expected to have lower pin-

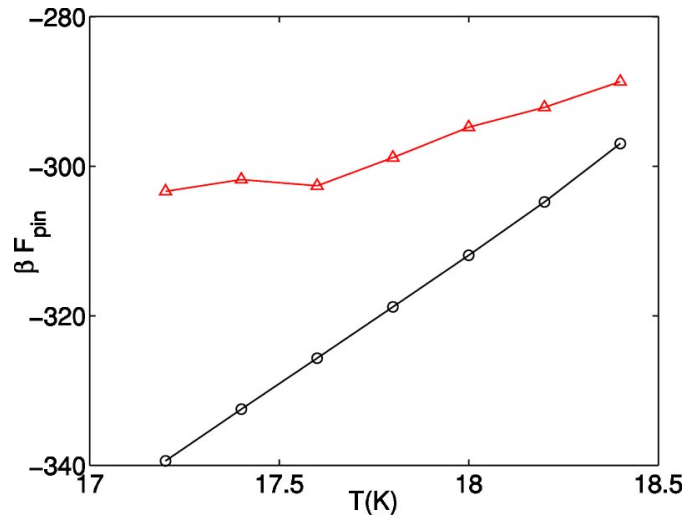

FIG. 10. (Color online) The pinning free energy (see text) averaged over five samples with $N_{v}=4096$ and $N_{p}=64$. Results for the $\mathrm{BrG}$ and $\mathrm{BoG}$ minima are shown by (red) triangles and (black) circles, respectively. The solid lines are straight lines joining the points.

ning energy but higher elastic energy than the BrG ones for the same pin configuration. The elastic energy dominates over the pinning energy at low temperatures in our low pinconcentration samples. This is why the BrG phase is globally stable at low $T$. Both these components of the free energy decrease in magnitude as the temperature is increased. The softening of the lattice near the melting transition causes the elastic energy to decrease faster than the pinning energy. This makes the pinning component of the free energy more important than the elastic part near $T_{m}^{0}$, thereby making the total free energy of the BoG minima (which, as discussed above, have lower pinning energy) lower than that of the $\mathrm{BrG}$ minima. The BoG to IL transition at a slightly higher temperature is driven by the usual entropic mechanism.

We can substantiate this qualitative explanation by evaluating the pinning component of the free energy, as defined in Eq. (2.4), for the BrG and BoG minima. The results of our calculation, averaged over five samples (each with 4096 vortices and 64 pins), are shown in Fig. 10. The pinning energy is negative for both kinds of minima, as expected. The absolute value of the pinning energy of the BoG minima shows a smooth (nearly linear) decrease as the temperature is increased. This is because both the value of the parameter $\Gamma$ [see Eq. (2.6)] that determines the depth of the pinning potential and the height of the local density peak at a pinning center decrease with increasing $T$. The plot for the $\mathrm{BrG}$ minima shows a similar but much slower behavior, still monotonic or nearly so: it is not clear whether the very shallow minimum near $T=17.6 \mathrm{~K}$ is significant. In some of the samples, the total number of occupied pinning centers increases by a small amount near this temperature, thereby reducing the value of the total pinning energy. This may reflect a better accommodation of the pinning centers by the lattice, which softens as the temperature is increased. This plot clearly demonstrates that the pinning centers are better accommodated at the BoG minima. This is in qualitative agreement with the results of Ref. 39, where it was argued that a state with no crystalline order gains more than a crystalline state from random point pinning. The difference in the pinning energies of the two kinds of minima decreases with 
increasing $T$, but the difference in the elastic component of the free energy decreases faster (this is clear from our results for the total free energy) due to a softening of the elastic constants with increasing temperature. The overall effect is a crossing of the free energies of the two kinds of minima near the melting transition.

Quantitatively, however, the values of the two parts of the free energy and their dependence on $T$ are determined by the material parameters of the superconductor and the properties of the pinning centers. Since several experimental studies $^{17-19}$ of the effects of irradiation-induced columnar pinning on the mixed state of BSCCO exist in the literature, we have used parameter values appropriate for this system. For other layered superconductors, the BoG phase might occur over a wider or narrower (even vanishing) range.

\section{Inhomogeneous melting of the vortex solid}

As mentioned in Sec. III B, the density distribution in both $\mathrm{BrG}$ and BoG minima is very inhomogeneous: at temperatures close to $T_{m}^{0}$ there are liquidlike regions, characterized by low values of the local peak densities in parts of the sample. In this subsection, we show that this inhomogeneity leads to a spatial variation of a "local melting temperature," defined below. As mentioned in the Introduction, such spatial variations of a local melting temperature have been deduced from measurements of the local magnetization in BSCCO samples with random point ${ }^{28}$ and columnar pinning. ${ }^{18}$

Since we are dealing with time-averaged densities, it is clear that the value of $\rho_{i}$ at a local peak of the density field provides a measure of the degree of localization of the vortex whose average position corresponds to the location of the density peak. A high (low) value of the local peak density implies strong (weak) localization. Smaller values of the local peak density imply mobile, liquidlike behavior. The value of the local density is, of course, equal to $\rho_{0}$ everywhere in the liquid state in the absence of pinning. The presence of pinning centers causes the local density to vary in space, but in the liquid state this variation does not lead to local peaks higher than about $3 \rho_{0}$ (excluding the vortices localized at the pinning centers). A similar result was also obtained in previous studies ${ }^{31}$ of vortices in the presence of pinning, where it was found that values of the peak density lower than about $3 \rho_{0}$ correspond to the liquid state. We therefore take the value $3 \rho_{0}$ of the local peak density as separating solid and liquidlike behaviors.

Using this criterion, we can determine whether a small region of the sample at a given minimum is in a locally "solid" or "liquid" state. To do this, we define a quantity $\rho_{a v}^{p}$ as the average of the local peak densities in a small region containing $\sim 100$ vortices (see Fig. 11). The very high local density peaks representing vortices trapped at pinning centers are not included in this average. Values of $\rho_{a v}^{p}$ substantially larger than $3 \rho_{0}$ indicate solidlike behavior in the region under consideration, while substantially lower values of $\rho_{a v}^{p}$ suggest a locally melted region. To determine how the local melting temperature varies from one part of the sample to another, we have studied the temperature dependence of $\rho_{a v}^{p}$ for different regions of the sample. Typical results are shown
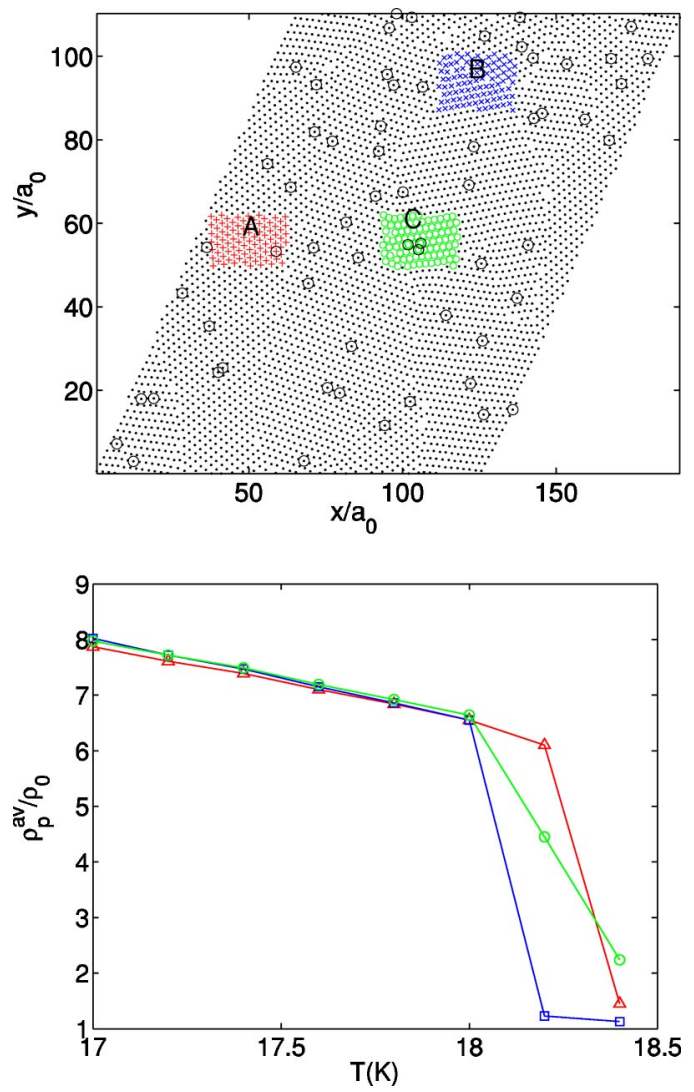

FIG. 11. (Color online) Determination of the local melting temperatures in different regions of a sample. The top panel, at $T$ $=18.0 \mathrm{~K}$ and for the BoG minimum, shows the three different regions ( $\mathrm{A}, \mathrm{B}$, and $\mathrm{C}$ ) considered in the calculation. The positions of local density peaks in these regions are indicated by (red) plus signs (region A), (blue) crosses (region B), and (green) circles (region C). The black dots represent the positions of the local density peaks in the other regions, and the (black) circles show the locations of the pinning centers. The bottom panel shows the dependence of the average local peak density $\rho_{a v}^{p}$ (normalized by the liquid density $\rho_{0}$ ) calculated for these regions on the temperature $T$. Data for regions $\mathrm{A}, \mathrm{B}$, and $\mathrm{C}$ are, respectively, shown by (red) triangles, (blue) squares, and (green) circles. The solid lines are guides to the eye.

in Fig. 11 for the pin configuration for which we have earlier shown detailed results in several figures. In the top panel, where the positions of the local density peaks in the BoG minimum at $T=18.0 \mathrm{~K}$ and the locations of the pinning centers are shown, we have indicated three regions for which the average local peak density $\rho_{a v}^{p}$ was calculated. The local density peaks in these regions are indicated by (red) plus signs (region A), (blue) crosses (region B), and (green) circles (region $\mathrm{C}$ ), while the black dots represent the positions of the local density peaks in the other regions. The bottom panel of Fig. 11 shows the dependence of the $\rho_{a v}^{p}$, s calculated for these three regions on the temperature $T$. At each value of $T$, the minimum with the lowest free energy at that temperature was used in the calculation of $\rho_{a v}^{p}$.

It is clear from the plot and from similar ones for other pin configurations (one of which was shown in Ref. 32) that the "local melting temperature," operationally defined as the temperature at which $\rho_{a v}^{p}$ drops from values well above $3 \rho_{0}$ 


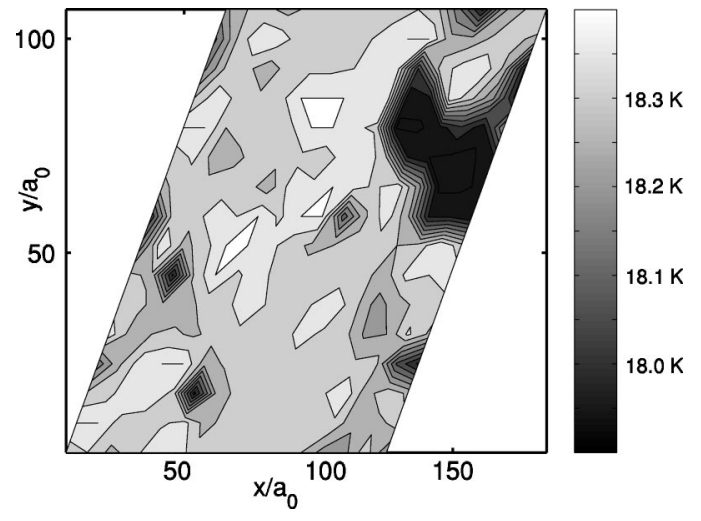

FIG. 12. Percolation and melting. Contour plot of the local melting temperature (as defined in the text), showing percolation of liquidlike regions at the melting transition. The spatial variation of the temperature at which local melting occurs in one of the samples with 4096 vortices and 64 pins is shown. The accompanying grayscale bar shows the correspondence between the local melting temperature and the gray-scale level: dark (light) regions in the plot correspond to low (high) values of the local melting temperature.

to values clearly below, varies from region to region. The range over which the local melting temperature varies is comparable to that found in the experiments. ${ }^{18,28}$ The values of the local melting temperature are strongly correlated with the local pin structure and the resulting defect structure of the vortex solid. Region B of the sample does not have any pinning center and is located across a grain boundary of the $\mathrm{BoG}$ minimum. As noted in Sec. III B, regions near grain boundaries appear to melt at temperatures that are slightly lower than the global transition temperature determined by the crossing of free energies. This is why the local melting temperature for region $\mathrm{B}$ is the lowest. This temperature corresponds to the transition from the $\mathrm{BrG}$ to the $\mathrm{BoG}$ state. In region $\mathrm{A}$ the vortices form a nearly perfect crystalline arrangement with no topological defects. The local melting temperature measured in this region is, as a consequence, close to that of the pure system. Region C contains a cluster of pinning centers, and the relatively large value of $\rho_{a v}^{p}$ for this region at temperatures higher than the BoG to IL transition temperature reflects the local solidlike structure of vortices situated near pinning centers. Thus, the spatial variation of the local transition temperature is closely correlated with the "pinning landscape" of the sample.

To illustrate further the spatial variation of the local melting temperature, we have shown in Fig. 12 a filled contour plot of the temperature at which local melting occurs in another sample with 4096 vortices and 64 pinning centers. To obtain this plot, we divided the sample into 256 equal-sized regions, each of which contains 16 vortices on the average, and calculated the average local peak density for each region. The minimum with the lowest free energy at each temperature was used in this calculation. The local melting temperature for each region was then defined to be the temperature at which the average local peak density in the region crosses the value $3 \rho_{0}$. In the filled contour plot of Fig. 12, dark (light) regions represent low (high) values of the local melting temperature, as indicated in the accompanying gray-scale bar. It is clear from the plot that the local melting temperature in this sample varies roughly between 17.9 and $18.4 \mathrm{~K}$. The overall upper melting temperature (BoG to IL) for this sample was found to be $18.3 \mathrm{~K}$. As in the case of the data shown in Fig. 11, we find that low values of the local melting temperature occur near grain boundaries in the BoG phase and high values occur near clusters of pinning centers.

We have found a second way of correlating the melting transition with the local density structure of the free energy minima. This second way is based on a previous study ${ }^{31}$ of the melting of the vortex lattice in the presence of periodic pinning, where it was found that the melting transition corresponds to the onset of percolation of liquidlike regions defined using the peak-density criterion mentioned above. The temperature at which liquidlike regions begin to percolate across the sample can be estimated from contour plots such as that shown in Fig. 12. For any given temperature, the regions in the plot that are darker than the gray-scale level corresponding to that temperature (as indicated in the accompanying gray-scale bar) exhibit melted (liquidlike) local structure, whereas lighter regions correspond to solidlike behavior. Thus, for each temperature, one has to examine whether regions that are darker than the gray-scale level corresponding to the temperature percolate across the sample.

We focus for this purpose on the upper transition between the BoG and IL phases. It is clear from Fig. 12 that regions that are darker than the gray-scale level corresponding to $18.3 \mathrm{~K}$ percolate across the sample. Thus, liquidlike regions percolate at this temperature. It is also clear that the liquidlike regions do not percolate at temperatures that are somewhat lower. This temperature closely agrees with the global transition temperature of $18.3 \mathrm{~K}$ found for this sample from the crossing of the free energies of the BoG and IL phases. Thus, we find, as previously, ${ }^{31}$ that the melting transition coincides with the onset of percolation of the liquidlike regions. Similar behavior was found in the other samples we have studied. This observation provides a convenient way of approximately locating the transition point. This may be useful in other situations where the method of locating the transition temperature from a crossing of free energies may not be easily implementable (e.g., at higher pin concentrations where the melting transition is expected to become continuous).

\section{CONCLUSIONS}

We have presented here the results of a detailed investigation of the structural and thermodynamic properties of a system of vortices in a highly anisotropic layered superconductor with a small concentration of randomly placed columnar pinning centers. Both the external magnetic field and the columnar pins are assumed to be perpendicular to the superconducting layers. Our method, based on numerical minimization of the appropriate free-energy functional, allows us to obtain very detailed information about the density distribution in the different free-energy minima (which allows us to reliably identify the phases corresponding to these minima), and to map out the phase diagram of the system.

There are several salient results of our study. The first one is the occurrence of a topologically ordered $\mathrm{BrG}$ phase at low 
temperatures. While the occurrence of a low-temperature $\mathrm{BrG}$ phase in superconductors with a low concentration of random point pinning centers is well-established now, relatively little is known about the existence of such a phase in superconductors with random columnar pins. Our results are consistent with those of a recent numerical study ${ }^{27}$ of a similar system. It is clear from our work that the $\mathrm{BrG}$ minima represent a phase distinct from the polycrystalline BoG phase also found in our study. We cannot conclusively rule out the possibility that free dislocations would appear at the nearly crystalline minima at length scales much longer than those considered in our numerical study. If this should happen, then the "hexatic glass" phase suggested in some earlier theoretical studies ${ }^{40}$ would become a possible candidate for describing the BrG minima found in our study. This phase, however, would be distinct from the BoG.

Our second important result is the occurrence of a twostep melting transition: we find that the low-temperature $\mathrm{BrG}$ phase transforms into a polycrystalline $\mathrm{BoG}$ phase as the temperature is increased, and this BoG phase then melts into the high-temperature IL at a slightly higher temperature. This conclusion about the occurrence of two distinct transitions would remain valid even if the true nature of the lowtemperature phase turns out to be slightly different from a Bragg glass: our work shows that the $\mathrm{BrG}$ and $\mathrm{BoG}$ minima are quite distinct from each other. The possibility of occurrence of a two-step melting transition of the vortex lattice in systems with random point pinning has been suggested earlier. ${ }^{14}$ Our work provides support to this suggestion. We have shown that the intermediate BoG phase arises because the pinning part of the free energy favors this polycrystalline phase over the nearly crystalline $\mathrm{BrG}$ phase. This result, obtained here for a highly anisotropic system with columnar pinning, is consistent with recent work ${ }^{39}$ on a less anisotropic system with point pinning, where good agreement between theory and experiment was obtained.

To our knowledge, the second (lower $T$ ) transition between the BoG and $\mathrm{BrG}$ phases has not been observed in experiments on layered superconductors with a small concentration of random columnar pins. This may be due to strong metastability: the BoG minimum into which the IL is expected to freeze as the temperature is decreased remains locally stable at temperatures lower than that at which its free energy crosses that of the BrG minimum, suggesting that it would be difficult to see in experiments the transition to the globally stable $\mathrm{BrG}$ phase. The situation here may be similar to that found in a recent experimental study ${ }^{41}$ of a low- $T_{c}$ superconductor with weak point pinning which is expected to exhibit a BrG phase at low $T$. It is, however, found in the experiment that the vortex solid obtained by cooling the sample in the presence of the external magnetic field has a polycrystalline structure, indicating that the metastability of this disordered state prevents the system from reaching the more ordered $(\mathrm{BrG})$ equilibrium state at low temperatures. In our numerical work, the BrG minima were obtained by performing the free-energy minimization from an initial configuration with crystalline order. It is not clear how a similar procedure can be adopted in experimental studies.

A low-temperature $\mathrm{BrG}$ phase has been observed in a recent simulation ${ }^{27}$ of a model of HTSC with a small concentration of columnar pinning centers. However, this simulation finds a single first-order transition between the BrG and IL phases at low pin concentrations: the small intermediate region of the BoG phase found in our study is not observed. This is probably due to the smallness of the system sizes $(\sim 100$ vortex lines) used in the simulation. As discussed in Sec. III C above, the polycrystalline BoG minima are found only if the sample size is larger than the typical size of the crystalline domains: only the $\mathrm{BrG}$ minimum is found at low temperatures if this condition is not satisfied. Since the crystalline domains become large at small pin concentrations, it is quite likely that a simulation with small system sizes and low pin concentration would not detect the BoG phase. Another possibility is that a narrow "two-phase" region found in Ref. 27 near the BrG melting transition actually corresponds to the intermediate BoG phase found in our study. A third possibility is that the intermediate $\mathrm{BoG}$ phase, which arises due to a competition between the elastic and pinning components of the free energy (see Sec. III C where it is shown that the pinning energy favors the BoG phase), does not appear in the simulation of Ref. 27 because the pins are assumed to be "weak" in that work.

Our study also illustrates the spatial inhomogeneity of the melting process in the presence of disorder: we have found that a "local" melting temperature defined using a criterion based on the degree of localization of the vortices shows considerable spatial variation. This spatial variation is strongly correlated with the "pinning landscape" associated with the random spatial location of the pinning centers. Our results about the spatial inhomogeneity of the local melting temperature are consistent with those of recent experiments ${ }^{18,28}$ on superconductors with pinning disorder.

These results establish the usefulness of our numerical method in dealing with the problem of vortex matter in the presence of random pinning. It would be interesting to examine the extent to which our results depend on the values of the parameters appearing in the free energy functional. It would also be clearly useful to carry out similar studies of other related problems, such as the complete phase diagram of systems with random columnar pins in the $T-c$ plane, and the phase behavior of systems with random point pinning. Some of these investigations are currently in progress.

\section{ACKNOWLEDGMENTS}

We thank G. I. Menon for several useful discussions. This work was supported in part by a joint grant from NSF (OISE-0352598) and DST (India). 
*Electronic address: cdgupta@physics.iisc.ernet in

†Electronic address: otvalls@umn.edu

${ }^{1}$ G. Blatter, M. V. Feigelman, V. B. Geshkenbein, A. I. Larkin, and V. M. Vinokur, Rev. Mod. Phys. 66, 1125 (1994).

${ }^{2}$ P. G. de Gennes, Superconductivity of Metals and Alloys (Addison-Wesley, Reading, MA, 1989).

${ }^{3}$ T. Nattermann, Phys. Rev. Lett. 64, 2454 (1990).

${ }^{4}$ T. Giamarchi and P. Le Doussal, Phys. Rev. B 52, 1242 (1995).

${ }^{5}$ T. Nattermann and S. Scheidl, Adv. Phys. 49, 607 (2000).

${ }^{6}$ T. Klein, I. Joumard, S. Blanchard, J. Marcus, R. Cubitt, T. Giamarchi, and P. Le Doussal, Nature (London) 413, 404 (2001).

${ }^{7}$ M. P. A. Fisher, Phys. Rev. Lett. 62, 1415 (1989).

${ }^{8}$ D. S. Fisher, M. P. A. Fisher, and D. A. Huse, Phys. Rev. B 43, 130 (1992).

${ }^{9}$ H. S. Bokil and A. P. Young, Phys. Rev. Lett. 74, 3021 (1995).

${ }^{10}$ A. Vestergren, J. Lidmar, and M. Wallin, Phys. Rev. Lett. 88, 117004 (2002).

${ }^{11}$ A. M. Petrean, L. M. Paulius, W.-K. Kwok, J. A. Fendrich, and G. W. Crabtree, Phys. Rev. Lett. 84, 5852 (2000) and references therein.

${ }^{12}$ D. R. Strachan, M. C. Sullivan, P. Fournier, S. P. Pai, T. Venkatesan, and C. J. Lobb, Phys. Rev. Lett. 87, 067007 (2001).

${ }^{13}$ S. S. Banerjee, T. V. C. Rao, A. K. Grover, M. J. Higgins, G. I. Menon, P. K. Mishra, D. Pal, S. Ramakrishnan, G. Ravikumar, V. C. Sahni, S. Sarkar, and C. V. Tomy, Physica C 355/1-2, 39 (2001), and references therein.

${ }^{14}$ G. I. Menon, Phys. Rev. B 65, 104527 (2002).

${ }^{15}$ L. Civale, A. D. Marwick, T. K. Worthington, M. A. Kirk, J. R. Thompson, L. Krusin-Elbaum, Y. Sun, J. R. Clem, and F. Holtzberg, Phys. Rev. Lett. 67, 648 (1991).

${ }^{16}$ R. C. Budhani, M. Suenaga, and S. H. Liou, Phys. Rev. Lett. 69, 3816 (1992).

${ }^{17}$ B. Khaykovich, M. Konczykowski, K. Teitelbaum, E. Zeldov, H. Shtrikman, and M. Rappaport, Phys. Rev. B 57, R14088 (1998).

${ }^{18}$ S. S. Banerjee, A. Soibel, Y. Myasoedov, M. Rappaport, E. Zeldov, M. Menghini, Y. Fasano, F. de la Cruz, C. J. van der Beek, M. Konczykowski, and T. Tamegai, Phys. Rev. Lett. 90, 087004 (2003).

${ }^{19}$ M. Menghini, Y. Fasano, F. de la Cruz, S. S. Banerjee, Y. Myasoedov, E. Zeldov, C. J. van der Beek, M. Konczykowski, and T.
Tamegai, Phys. Rev. Lett. 90, 147001 (2003).

${ }^{20}$ D. R. Nelson and V. M. Vinokur, Phys. Rev. B 48, 13060 (1993).

${ }^{21}$ L. Radzihovsky, Phys. Rev. Lett. 74, 4923 (1995).

${ }^{22}$ A. I. Larkin and V. M. Vinokur, Phys. Rev. Lett. 75, 4666 (1995).

${ }^{23}$ A. V. Lopatin and V. M. Vinokur, Phys. Rev. Lett. 92, 067008 (2004).

${ }^{24}$ C. Wengel and U. C. Tauber, Phys. Rev. B 58, 6565 (1988).

${ }^{25}$ P. Sen, N. Trivedi, and D. M. Ceperley, Phys. Rev. Lett. 86, 4092 (2001).

${ }^{26}$ S. Tyagi and Y. Y. Goldschmidt, Phys. Rev. B 67, 214501 (2003).

${ }^{27}$ Y. Nonomura and $\mathrm{X}$. Hu, cond-mat/0212609 (unpublished).

${ }^{28}$ A. Soibel, Y. Myasoedov, M. L. Rappaport, T. Tamegai, S. S. Banerjee, and E. Zeldov, Phys. Rev. Lett. 87, 167001 (2001).

${ }^{29}$ T. V. Ramakrishnan and M. Yussouff, Phys. Rev. B 19, 2775 (1979).

${ }^{30}$ C. Dasgupta and O. T. Valls, Phys. Rev. Lett. 87, 257002 (2001).

${ }^{31}$ C. Dasgupta and O. T. Valls, Phys. Rev. B 66, 064518 (2002).

${ }^{32}$ Some earlier results on this important point are in: C. Dasgupta and O. T. Valls, Phys. Rev. Lett. 91, 127002 (2003).

${ }^{33}$ S. Sengupta, C. Dasgupta, H. R. Krishnamurthy, G. I. Menon, and T. V. Ramakrishnan, Phys. Rev. Lett. 67, 3444 (1991).

${ }^{34}$ G. Menon, C. Dasgupta, H. R. Krishnamurthy, T. V. Ramakrishnan, and S. Sengupta, Phys. Rev. B 54, 16192 (1996).

${ }^{35}$ Detailed comparisons between the results of density-functional calculations for the pure system and experimental results for BSCCO may be found in Ref. 34 and in P. S. Cornaglia and C. A. Balseiro, Phys. Rev. B 61, 784 (2000). Quantities compared include the shape and position of the first-order melting line in the $B-T$ plane, and the magnitude and temperature dependence of the discontinuities in magnetic induction and entropy at the transition.

${ }^{36}$ See, e.g., J. P. Hansen and I. R. McDonald, Theory of Simple Liquids (Academic, London, 1986).

${ }^{37}$ C. Dasgupta and D. Feinberg, Phys. Rev. B 57, 11730 (1998).

${ }^{38}$ C. Dasgupta, Europhys. Lett. 20, 131 (1992).

${ }^{39}$ D. Li and B. Rosenstein, Phys. Rev. Lett. 90, 167004 (2003).

${ }^{40}$ E. M. Chudnovsky, Phys. Rev. B 43, 7831 (1991).

${ }^{41}$ Y. Fasano, M. Menghini, F. de la Cruz, Y. Paltiel, Y. Myasoedov, E. Zeldov, M. J. Higgins, and S. Bhattacharya, Phys. Rev. B 66, 020512(R) (2002). 